\title{
THE $\mathbb{Q}$-KORSELT SET OF pq
}

\author{
NEJIB GHANMI
}

\begin{abstract}
Let $N$ be a positive integer, $\mathbb{A}$ be a nonempty subset of $\mathbb{Q}$ and $\alpha=\frac{\alpha_{1}}{\alpha_{2}} \in \mathbb{A} \backslash\{0, N\}$. $\alpha$ is called an $N$-Korselt base (equivalently $N$ is said an $\alpha$-Korselt number) if $\alpha_{2} p-\alpha_{1}$ is a divisor of $\alpha_{2} N-\alpha_{1}$ for every prime $p$ dividing $N$. The set of all Korselt bases of $N$ in $\mathbb{A}$ is called the $\mathbb{A}$-Korselt set of $N$ and is simply denoted by $\mathbb{A}-\mathcal{K} \mathcal{S}(N)$.

Let $p$ and $q$ be two distinct prime numbers. In this paper, we study the $\mathbb{Q}$-Korselt bases of $p q$, where we give in detail how to provide $\mathbb{Q}-\mathcal{K} \mathcal{S}(p q)$. Consequently, we finish the incomplete characterization of the Korselt set of $p q$ over $\mathbb{Z}$ given in [4, by supplying the set $\mathbb{Z}-\mathcal{K} \mathcal{S}(p q)$ when $q<2 p$.
\end{abstract}

\section{INTRODUCTION}

The notion of Korselt numbers ( or $\alpha$-Korselt number with $\alpha \in \mathbb{Z}$ ) was introduced by Bouallègue-Echi-Pinch [2, 7] as a generalization of Carmichael numbers [1, 3]. Korselt numbers are defined simply as numbers which meet a generalized Korselt criterion as follows.

Definition 1.1. 8] Let $\alpha \in \mathbb{Z} \backslash\{0\}$. A positive integer $N$ is said to be an $\alpha$-Korselt number ( $K_{\alpha}$-number, for short) if $N \neq \alpha$ and $p-\alpha$ divides $N-\alpha$ for each prime divisor $p$ of $N$.

Considerable progress has been made investigating Korselt numbers last years spatially in [2, 4, 5, 7]. Many properties of Carmichael numbers are extended for Korselt numbers. However, many related questions remain open until now, such as the infinitude of Korselt numbers, providing a complete characterization of the Korselt set of such a number, etc. Recently, Ghanmi proposed in [5, 6] another generalization of Carmichael numbers; he extended the notion of Korselt numbers to $\mathbb{Q}$ by stating the following definitions.

2010 Mathematics Subject Classification. Primary 11Y16; Secondary $11 Y 11$, $11 A 51$.

Key words and phrases. Prime number, Carmichael number, Square free composite number, Korselt base, Korselt number, Korselt set. 
Definition 1.2. [5]Let $N \in \mathbb{N} \backslash\{0,1\}, \alpha=\frac{\alpha_{1}}{\alpha_{2}} \in \mathbb{Q} \backslash\{0\}$ and $\mathbb{A}$ be a subset of $\mathbb{Q}$. Then

(1) $N$ is said to be an $\alpha$-Korselt number ( $K_{\alpha}$-number, for short), if $N \neq \alpha$ and $\alpha_{2} p-\alpha_{1}$ divides $\alpha_{2} N-\alpha_{1}$ for every prime divisor $p$ of $N$.

(2) By the $\mathbb{A}$-Korselt set of the number $N$ (or the Korselt set of $N$ over $\mathbb{A})$, we mean the set $\mathbb{A}-\mathcal{K} \mathcal{S}(N)$ of all $\beta \in \mathbb{A} \backslash\{0, N\}$ such that $N$ is a $K_{\beta}$-number.

(3) The cardinality of $\mathbb{A}-\mathcal{K} \mathcal{S}(N)$ will be called the $\mathbb{A}$-Korselt weight of $N$; we denote it by $\mathbb{A}-\mathcal{K} \mathcal{W}(N)$.

It's obvious by this definition, that for $\alpha \in \mathbb{Z} \backslash\{0\}$ (i.e $\alpha_{2}=1$ ) we obtain the original $\alpha$-Korselt numbers introduced by Bouallègue-EchiPinch [2].

Definition 1.3. [6]Let $N \in \mathbb{N} \backslash\{0,1\}, \alpha \in \mathbb{Q}$ and $\mathbb{B}$ be a subset of $\mathbb{N}$. Then

(1) $\alpha$ is called $N$-Korselt base $\left(K_{N}\right.$-base, for short), if $N$ is a $K_{\alpha^{-}}$ number.

(2) By the $\mathbb{B}$-Korselt set of the base $\alpha$ (or the Korselt set of the base $\alpha$ over $\mathbb{B})$, we mean the set $\mathbb{B}-\mathcal{K} \mathcal{S}(B(\alpha))$ of all $M \in \mathbb{B}$ such that $\alpha$ is a $K_{M}$-base.

(3) The cardinality of $\mathbb{B}-\mathcal{K S}(B(\alpha))$ will be called the $\mathbb{B}$-Korselt weight of the base $\alpha$; we denote it by $\mathbb{B}-\mathcal{K} \mathcal{W}(B(\alpha))$.

The set of all $\alpha$-Korselt numbers when $\alpha$ varies in $\mathbb{Q}$, is called the $\mathbb{Q}$-Korselt numbers (or rational Korselt numbers or the set of Korselt numbers over $\mathbb{Q}$ ). The set of all $N$-Korselt bases in $\mathbb{Q}$ when $N$ varies in $\mathbb{N}$, is called the $\mathbb{N}$-Korselt bases in $\mathbb{Q}$ (or $\mathbb{N}$-Korselt rational bases or the set of Korselt rational bases over $\mathbb{N}$ ).

As we know, it's not easy in general to determine the Korselt set of a given number $N$ over $\mathbb{Z}$; even for the simple case when $N=p q$ with $p$ and $q$ are two distinct prime numbers. This difficulty is mentioned in 4 by Ghanmi and Echi, where they characterized the $\mathbb{Z}$-Korselt set of $p q$ and left the case when $q<2 p$ without solution. Fortunately, this problem will be solved in our present work; in fact, the set $\mathbb{Z}-\mathcal{K} \mathcal{S}(p q)$ when $q<2 p$, will be completely determined after studying the Korselt set of $p q$ over $\mathbb{Q}$.

In this paper, and for given distinct prime numbers $p$ and $q$, we will discuss in Section 2, the belonging sets of $\alpha_{1}$ and $\alpha_{2}$ in $\mathbb{Z}$ for which $\alpha=\frac{\alpha_{1}}{\alpha_{2}}$ is a Korselt rational base of $p q$. In Section 3 and by some results given in Section 2, we will characterize completely the Korselt 
rational set of $p q$. Furthermore, this allowed us to deduce immediately the $\mathbb{Z}$-Korselt set of $p q$ when $q<2 p$.

For all the following let $\alpha=\frac{\alpha_{1}}{\alpha_{2}} \in \mathbb{Q}, p<q$ be two primes, $N=p q$ and $i, j, s, t$ be the integers given by the Euclidean divisions of $q$ and $\alpha_{1}$ by $p: q=i p+s$ and $\alpha_{1}=j p+t$ with $s \in\{1, \ldots, p-1\}, t \in$ $\{0,1, \ldots, p-1\}$.

For $\alpha=\frac{\alpha_{1}}{\alpha_{2}} \in \mathbb{Q}$, we will suppose without loss of generality that $\alpha_{2} \in \mathbb{N} \backslash\{0\}, \alpha_{1} \in \mathbb{Z}$ and $\operatorname{gcd}\left(\alpha_{1}, \alpha_{2}\right)=1$.

As the case of $\alpha \in \mathbb{Z}$ (i.e. $\alpha_{2}=1$ ) is discussed in [4], our attention will center in all this paper, only on the case when $\alpha_{2} \geq 2$.

\section{Properties of $p q$-Korselt Rational BASES}

Proposition 2.1. [5] Let $\alpha=\frac{\alpha_{1}}{\alpha_{2}} \in \mathbb{Q}$ such that $\operatorname{gcd}\left(\alpha_{1}, N\right)=1$. If $\alpha$ is a $K_{N}$-base, then the following inequalities hold.

$$
q-p+1 \leq \alpha \leq q+p-1 \text {. }
$$

Now, we give informations about the Korselt set of $p q$ over $\mathbb{Q}$ when $\operatorname{gcd}\left(\alpha_{1}, N\right) \neq 1$.

Proposition 2.2. [5, Proposition 2.4] Suppose that $N$ is a $K_{\alpha}$-number with $\operatorname{gcd}\left(\alpha_{1}, N\right) \neq 1$. Then the following assertions hold.

1) If $\alpha \in \mathbb{Z}\left(\right.$ i.e. $\left.\alpha_{2}=1 ; \alpha=\alpha_{1}\right)$, then $q \nmid \alpha, \quad p \mid \alpha$ and

$$
\alpha \in\left\{\left\lfloor\frac{q}{p}\right\rfloor p,\left\lceil\frac{q}{p}\right\rceil p\right\} .
$$

2) If $\alpha \in \mathbb{Q} \backslash \mathbb{Z}$, then $\frac{q}{p} \leq \alpha \leq q+p-1$.

Proposition 2.3. Let $\alpha=\frac{\alpha_{1}}{\alpha_{2}} \in \mathbb{Q}$ be such that $\operatorname{gcd}\left(\alpha_{1}, N\right)=1$.

If $\alpha$ is a $K_{N}$-base, then the following properties hold.

(1) If $q<2 p$, then $1 \leq \alpha_{2} \leq p$.

(2) If $2 p<q<3 p$, then $1 \leq \alpha_{2}<3$.

(3) If $3 p<q<4 p$, then $1 \leq \alpha_{2}<2$.

(4) If $q>4 p$, then $\alpha_{2}=1$ (i.e. $\alpha \in \mathbb{Z}$ ).

Proof. By definition, $\alpha$ is a $K_{N}$-base if and only if

$$
\left\{\begin{array}{c|c}
\alpha_{2} p-\alpha_{1} & p(q-1) \\
\alpha_{2} q-\alpha_{1} & q(p-1)
\end{array}\right.
$$


Since $\operatorname{gcd}\left(\alpha_{1}, p\right)=\operatorname{gcd}\left(\alpha_{1}, q\right)=1$ and $q=i p+s,\left(S_{1}\right)$ is equivalent to

$\left(S_{2}\right) \quad\left\{\begin{array}{l|l}\alpha_{2} p-\alpha_{1} & q-1=i p+s-1 \\ \alpha_{2}(i p+s)-\alpha_{1} & p-1\end{array}\right.$

This implies that

$$
-\alpha_{2} p+\alpha_{1} \leq i p+s-1
$$

and

$$
\alpha_{2}(i p+s)-\alpha_{1} \leq p-1 \text {. }
$$

The sum of (2.1) and (2.2) gives

$$
\alpha_{2}((i-1) p+s) \leq(i+1) p+s-2,
$$

as $\alpha_{2} \geq 1$, it follows that

$$
\alpha_{2}(i-1) p \leq(i+1) p+s\left(1-\alpha_{2}\right)-2<(i+1) p .
$$

Two cases are to be discussed.

a) If $q<2 p$ hence $q=p+s$ with $s \geq 2$, (since $p=2 ; q=3 ; s=1$ and $\alpha_{2}=3$ would imply that $\left.2 \mid \alpha_{1}\right)$, then by (2.3) we get

$$
1 \leq \alpha_{2} \leq \frac{2 p-2}{s}+1 \leq p
$$

b) Suppose that $i \geq 2$. Then by (2.4), we obtain $1 \leq \alpha_{2}<\frac{i+1}{i-1}$.

Hence, the following subcases hold.

- If $2 p<q<3 p$ (i.e. $i=2$ ), then $\alpha_{2}<3$.

- If $3 p<q<4 p$ (i.e. $i=3$ ), then $\alpha_{2}<2$.

- If $q>4 p$ (i.e. $i \geq 4$ ), then $\alpha_{2}=1$.

Each case in Proposition 2.3 will be discussed separately in the following two results.

Proposition 2.4. Let $\alpha=\frac{\alpha_{1}}{\alpha_{2}} \in \mathbb{Q}$ be such that $\operatorname{gcd}\left(\alpha_{1}, N\right)=1$. If $\alpha$ is a $K_{N}$-base with $q>2 p$, then $\alpha \in \mathbb{Z}$.

Proof. Let $\alpha=\frac{\alpha_{1}}{\alpha_{2}} \in \mathbb{Q}-\mathcal{K S}(N)$. First, let us note that if $i \geq 3$, then by Proposition 2.3 we have $\alpha \in \mathbb{Z}$. Hence, we may assume that $i=2$.

Substituting $\alpha_{1}=j p+t$ in $\left(S_{2}\right)$, we get

$$
\left\{\begin{array}{l|l}
\left(\alpha_{2}-j\right) p-t & i p+s-1 \\
\left(\alpha_{2} i-j\right) p+\alpha_{2} s-t & p-1
\end{array}\right.
$$

which implies that 


$$
\left\{\begin{array}{l}
\left(j-\alpha_{2}-i\right) p \leq s-t-1 \\
\alpha_{2} s-t+1 \leq\left(j-\alpha_{2} i+1\right) p
\end{array}\right.
$$

As $s-t-1 \leq p-3$ and $-p+\alpha_{2}+2 \leq \alpha_{2} s-t+1$, it follows that

$$
\left\{\begin{array}{l}
\left(j-\alpha_{2}-i\right) p \leq p-3 \\
-p+\alpha_{2}+2 \leq\left(j-\alpha_{2} i+1\right) p
\end{array}\right.
$$

hence

$$
\left(S_{4}\right) \quad \begin{cases}j & \leq \alpha_{2}+i \\ \alpha_{2} i-1 & \leq j\end{cases}
$$

Now, let us show that for $i=2$, we have $\alpha_{2}=1$ (i.e. $\alpha \in \mathbb{Z}$ ). If $i=2$, then $\alpha_{2}<3$ by Proposition 2.3. Suppose that $\alpha_{2}=2$. Then by $\left(S_{4}\right)$, $j=3$ or $j=4$.

- If $j=3$, then $\left(S_{3}\right)$ gives

$$
\left\{\begin{array}{l|l}
p+t \quad \mid 2 p+s-1 \\
p+2 s-t \mid p-1
\end{array}\right.
$$

By (2.5) we must have $2 p+s-1=2(p+t)$, hence $s=2 t+1$. Therefore $p+2 s-t=p+3 t+2>p-1$, which contradicts (2.6).

- If $j=4$, then $\left(S_{3}\right)$ becomes

$$
\left\{\begin{array}{l}
2 p+t \mid 2 p+s-1 \\
2 s-t \mid p-1
\end{array}\right.
$$

By (2.7) we must have $2 p+t=2 p+s-1$, so that $t=s-1$. As $q=2 p+s$, then $s$ is odd and so $\alpha_{1}=j p+t=4 p+s-1$ is even. But, since $\alpha_{2}=2$, we get a contradiction with the fact that $\operatorname{gcd}\left(\alpha_{1}, \alpha_{2}\right)=1$

Finally, we conclude that $\alpha_{2} \neq 2$. So, $\alpha_{2}=1$.

Proposition 2.5. Let $\alpha=\frac{\alpha_{1}}{\alpha_{2}} \in \mathbb{Q}$ be such that $\operatorname{gcd}\left(\alpha_{1}, N\right)=1$. If $\alpha$ is a $K_{N}$-base with $q<2 p$, then $\alpha_{2} \in\{j-1, j, j+1\}$.

Proof. If $\alpha$ is a $K_{N}$-base such that $\operatorname{gcd}\left(\alpha_{1}, p\right)=\operatorname{gcd}\left(\alpha_{1}, q\right)=1, q=p+s$ and $\alpha_{1}=j p+t$, then by $\left(S_{2}\right)$ we get

Hence

$$
\left(S_{5}\right) \quad\left\{\begin{array}{l|l}
\left(\alpha_{2}-j\right) p-t & p+s-1 \\
\left(\alpha_{2}-j\right) p+\alpha_{2} s-t & p-1
\end{array}\right.
$$

$$
\begin{cases}-p-s+1 \leq\left(\alpha_{2}-j\right) p-t & \leq p+s-1 \\ -p+1 & \leq\left(\alpha_{2}-j\right) p+\alpha_{2} s-t \leq p-1\end{cases}
$$


so that

$$
\left\{\begin{array}{l}
-p-s+t+1 \leq\left(\alpha_{2}-j\right) p \leq p+s+t-1 \\
-p-\alpha_{2} s+t+1 \leq\left(\alpha_{2}-j\right) p \leq p-\alpha_{2} s+t-1
\end{array}\right.
$$

This implies that

$$
-p-s+t+1 \leq\left(\alpha_{2}-j\right) p \leq p-\alpha_{2} s+t-1
$$

Since, in addition

$$
-2 p+3=-p-(p-1)+1+1 \leq-p-s+t+1
$$

and

$$
p-\alpha_{2} s+t-1 \leq p-1+p-1-1=2 p-3,
$$

it follows that

$$
\left\{\begin{aligned}
-2 p<-2 p+3 & \leq\left(\alpha_{2}-j\right) p \\
\left(\alpha_{2}-j\right) p & \leq 2 p-3<2 p
\end{aligned}\right.
$$

hence

$$
-2<\alpha_{2}-j<2 .
$$

So, we deduce that $\alpha_{2} \in\{j-1, j, j+1\}$.

The previous proposition leads us to discuss separately in the next three lemmas, each case of $\alpha_{2} \in\{j-1, j, j+1\}$ in order to fully determine the $K_{N}$-base $\alpha$. For the rest of this paper, let us define for an integer $m$ the set $\operatorname{Div}(m-1)=\left\{d_{m} \in \mathbb{N}^{*} ; d_{m} \mid(m-1)\right\}$.

Lemma 2.6. Let $\alpha=\frac{\alpha_{1}}{\alpha_{2}} \in \mathbb{Q}$ be such that $\operatorname{gcd}\left(\alpha_{1}, N\right)=1$. Suppose that $q<2 p, j \geq 2$ and $\alpha_{1}=\left(\alpha_{2}+1\right) p+t$ (i.e. $\left.\alpha_{2}=j-1\right)$. Then the following assertions hold.

(1) If $\alpha$ is a $K_{N}$-base then $\alpha_{2}$ is odd and $1 \leq \alpha_{2} \leq p$.

(2) $\alpha$ is a $K_{N}$-base if and only if there exist $\varepsilon \in\{-1,1\}$ and an even integer $d_{p} \in \operatorname{Div}(p-1)$ such that $t=s-1$ and $\alpha_{2}=\frac{q-1-\varepsilon d_{p}}{s}$.

Proof. (1) First, let us show that $p \neq 2$. Since $\alpha$ is a $K_{N^{-}}$base with $\operatorname{gcd}\left(\alpha_{1}, p\right)=\operatorname{gcd}\left(\alpha_{1}, q\right)=1, q=p+s$ and $\alpha_{1}=\left(\alpha_{2}+1\right) p+t$, we get by $\left(S_{5}\right)$

$$
\left(S_{6}\right) \quad \begin{cases}p+t & \mid p+s-1 \\ p+t-\alpha_{2} s & \mid p-1\end{cases}
$$

By (2.9) we must have $p+t=p+s-1$, hence $t=s-1$. 
Suppose by contradiction that $p=2$. As $1 \leq s \leq p-1=1$, it follows that $s=1$ hence $t=0$ and so $\alpha_{1}=\left(\alpha_{2}+1\right) p$. Therefore, $p=2 \mid \alpha_{1}$, which contradicts the fact that $\operatorname{gcd}\left(\alpha_{1}, p\right)=1$.

Now, since $p \geq 3$ and $q=p+s$, it follows that $s$ is even and so $t$ is odd. Further, we claim that $\alpha_{2}$ is odd, else if $\alpha_{2}$ is even and as $t$ is odd then $\alpha_{1}=\left(\alpha_{2}+1\right) p+t$ will be even, hence $2 \mid \operatorname{gcd}\left(\alpha_{1}, \alpha_{2}\right)=1$, which is impossible.

Proposition 2.3 (1) implies that $1 \leq \alpha_{2} \leq p$.

(2) Since $p \geq 3$ and $s$ is even, we obtain by $\left(S_{6}\right)$ the equivalence: $\alpha \in \mathbb{Q}-\mathcal{K S}(N)$ if and only if there exist $\varepsilon \in\{-1,1\}$ and an even integer $d_{p} \in \operatorname{Div}(p-1)$ such that

$$
\left\{\begin{array}{l}
t=s-1 \\
p-1-\left(\alpha_{2}-1\right) s=\varepsilon d_{p}
\end{array}\right.
$$

This is equivalent to $t=s-1$ and $\alpha_{2}=\frac{q-1-\varepsilon d_{p}}{s}$.

Lemma 2.7. Let $\alpha=\frac{\alpha_{1}}{\alpha_{2}} \in \mathbb{Q}$ be such that $\operatorname{gcd}\left(\alpha_{1}, N\right)=1$. Suppose that $q<2 p, j \geq 1$ and $\alpha_{1}=\alpha_{2} p+t \quad$ (i.e. $\alpha_{2}=j$ ). Then, $\alpha$ is a $K_{N}$-base if and only if there exist $\varepsilon \in\{-1,1\}, d_{p} \in \operatorname{Div}(p-1)$ and $d_{q} \in \operatorname{Div}(q-1)$ such that $t=d_{q} \leq p-1$ and $\alpha_{2}=\frac{d_{q}+\varepsilon d_{p}}{s}>0$.

Proof. Suppose that $\alpha \in \mathbb{Q}-\mathcal{K} \mathcal{S}(N)$ with $\operatorname{gcd}\left(\alpha_{1}, p\right)=\operatorname{gcd}\left(\alpha_{1}, q\right)=1$, $\alpha_{1}=\alpha_{2} p+t$ and $q=p+s$. Then $\left(S_{5}\right)$ becomes

$$
\left\{\begin{array}{l|l}
t & q-1 \\
\alpha_{2} s-t & p-1
\end{array}\right.
$$

It follows that, $\alpha$ is a $K_{N}$-base if and only if there exist $\varepsilon \in\{-1,1\}$, $d_{p} \in \operatorname{Div}(p-1)$ and $d_{q} \in \operatorname{Div}(q-1)$ such that

$$
\left\{\begin{array}{l}
t=d_{q} \\
\alpha_{2} s-t=\varepsilon d_{p}
\end{array}\right.
$$

which is equivalent to $t=d_{q}$ and $\alpha_{2}=\frac{d_{q}+\varepsilon d_{p}}{s}>0$.

Lemma 2.8. Let $\alpha=\frac{\alpha_{1}}{\alpha_{2}} \in \mathbb{Q}$ be such that $\operatorname{gcd}\left(\alpha_{1}, N\right)=1$. Suppose that $q<2 p$ and $\alpha_{1}=\left(\alpha_{2}-1\right) p+t \quad$ (i.e. $\left.\alpha_{2}=j+1\right)$. Then, $\alpha$ is a $K_{N}$-base if and only if there exist $d_{p} \in \operatorname{Div}(p-1), d_{p} \geq 2$ and $d_{q} \in \operatorname{Div}(q-1)$ such that $t=p-d_{q}>0$ and $\alpha_{2}=\frac{d_{p}-d_{q}}{s}>0$. 
Proof. If $\alpha \in \mathbb{Q}-\mathcal{K S}(N)$ such that $\operatorname{gcd}\left(\alpha_{1}, p\right)=\operatorname{gcd}\left(\alpha_{1}, q\right)=1, q=p+s$ and $\alpha_{1}=\left(\alpha_{2}-1\right) p+t$, then $\left(S_{5}\right)$ becomes

$$
\left\{\begin{array}{l|l}
p-t & q-1 \\
p-t+\alpha_{2} s & p-1
\end{array}\right.
$$

Therefore, $\alpha$ is a $K_{N}$-base if and only if there exist $d_{p} \in \operatorname{Div}(p-1)$ and $d_{q} \in \operatorname{Div}(q-1)$ such that

$$
\left\{\begin{array}{l}
p-t=d_{q} \\
\alpha_{2} s+p-t=d_{p} \geq 2
\end{array}\right.
$$

this is equivalent to $t=p-d_{q}>0$ and $\alpha_{2}=\frac{d_{p}-d_{q}}{s}>0$.

By the next result, we give bounds for $\alpha_{2}$ when $\alpha=\frac{\alpha_{1}}{\alpha_{2}}$ is a $K_{N^{-}}$base where $\operatorname{gcd}\left(\alpha_{1}, N\right)=q$.

Proposition 2.9. Let $\alpha=\frac{\alpha_{1}}{\alpha_{2}} \in \mathbb{Q}-\mathcal{K S}(N)$ be such that $\operatorname{gcd}\left(\alpha_{1}, N\right)=$ q. Then the following assertions hold.

(1) If $q<2 p$, then $1 \leq \alpha_{2} \leq \frac{p(p+2)}{2}$.

(2) If $q>2 p$, then $1 \leq \alpha_{2}<\frac{p(i+1)}{i-1}$.

Proof. Suppose that $\alpha \in \mathbb{Q}$ - $\mathcal{K S}(N)$ such that $\operatorname{gcd}\left(\alpha_{1}, N\right)=q$, so that $\operatorname{gcd}\left(\alpha_{1}, p\right)=1$. Then, by $\left(S_{1}\right)$ we get

$$
\left\{\begin{array}{l|l}
\alpha_{2} p-\alpha_{1} & q-1 \\
\alpha_{2} q-\alpha_{1} & q(p-1)
\end{array}\right.
$$

Therefore

$$
\left\{\begin{array}{l}
-\alpha_{2} p+\alpha_{1} \leq q-1 \\
\alpha_{2} q-\alpha_{1} \leq q(p-1)
\end{array}\right.
$$

hence, by summing (2.11) and (2.12) we get

$$
\alpha_{2}(q-p) \leq q p-1<q p .
$$

Two cases are to be discussed.

(1) If $i=1$, then $q=p+s$. We distinguish two subcases.

- If $s$ is even (i.e $s \geq 2$ ), then by (2.13) we obtain

$$
\alpha_{2} s=\alpha_{2}(q-p)<q p=(p+s) p .
$$


As $s \geq 2$, it follows that

$$
\alpha_{2}<\frac{(p+s) p}{s} \leq \frac{(p+2) p}{2} .
$$

- If $s$ is odd (i.e $s=1, q=3$ and $p=2$ ), then easily we can prove that $\alpha \in\left\{\frac{3}{2}, \frac{9}{4}\right\}$, and so $\alpha_{2} \leq \frac{(p+2) p}{2}$.

(2) If $q=i p+s>2 p$, then by (2.13) we get

$$
\begin{aligned}
& \alpha_{2}(i-1) p<\alpha_{2}((i-1) p+s)=\alpha_{2}(q-p)<q p<(i+1) p^{2} . \\
& \text { hence } \alpha_{2}<\frac{(i+1) p}{i-1} .
\end{aligned}
$$

Now, by the following two results, we determine all $K_{N}$-bases $\alpha \in \mathbb{Q}$ such that $\operatorname{gcd}\left(\alpha_{1}, N\right)=q$ and $q<2 p$.

Proposition 2.10. Let $\alpha=\frac{\alpha_{1}}{\alpha_{2}} \in \mathbb{Q}-\mathcal{K S}(N)$ such that $\operatorname{gcd}\left(\alpha_{1}, N\right)=q$. Suppose that $\alpha_{1}=\alpha_{1}^{\prime} q$ and $q<2 p$. Then the following assertions hold.

(1) $\alpha_{1}^{\prime}<\alpha_{2}$.

(2) If $\alpha_{1}^{\prime}=1$ then $\alpha_{2} \in\{2,3\}$, both for $p=3$; $q=5$, otherwise we have

(a) $\alpha_{2}=2$ if and only if $(p-s) \mid(2 s-1)$.

(b) $\alpha_{2}=3$ if and only if $p>2$ and $s=\frac{p+1}{2}$.

Proof. (1) Let $\alpha=\frac{\alpha_{1}}{\alpha_{2}} \in \mathbb{Q}-\mathcal{K} \mathcal{S}(N)$ be such that $\operatorname{gcd}\left(\alpha_{1}, p\right)=1$, $\alpha_{1}=\alpha_{1}^{\prime} q$ and $q=p+s$. Then $\left(S_{1}\right)$ is equivalent to

$$
\left(S_{7}\right) \quad \begin{cases}\left(\alpha_{2}-\alpha_{1}^{\prime}\right) p-\alpha_{1}^{\prime} s & \mid p+s-1 \\ \alpha_{2}-\alpha_{1}^{\prime} & \mid p-1\end{cases}
$$

First, by (2.16), we have $\alpha_{1}^{\prime} \neq \alpha_{2}$. Now, we claim that $\alpha_{1}^{\prime}<$ $\alpha_{2}$. If is not the case and as $\alpha_{1}^{\prime}>0$, then we get $\left(\alpha_{1}^{\prime}-\alpha_{2}\right) p+$ $\alpha_{1}^{\prime} s \geq p+s>0$, which contradicts (2.15).

(2) Suppose that $\alpha_{1}^{\prime}=1$. Then by (2.15) we obtain

$$
\left(\alpha_{2}-1\right) p-s \leq p+s-1 \text {. }
$$

As $s \leq p-1$, it follows that $\left(\alpha_{2}-2\right) p \leq 2 s-1<2 p$, hence $\alpha_{2}<4$. But, since $\alpha_{2}>\alpha_{1}^{\prime}=1$, it yields that $\alpha_{2} \in\{2,3\}$.

(a) If $\alpha_{2}=2$, then since $\alpha_{1}^{\prime}=1,\left(S_{7}\right)$ will be reduced to $(p-s) \mid(p+s-1)=p-s+2 s-1$, which is equivalent to $(p-s) \mid(2 s-1)$. 
(b) Now, suppose that $\alpha_{2}=3$. Then $\left(S_{7}\right)$ is simply reduced to $2 \mid p-1$ and $(2 p-s) \mid(p+s-1)$. Since $p+s-1<2(2 p-s)$, it follows that $\left(S_{7}\right)$ is equivalent to $p>2$ and $2 p-s=p+s-1$, so $s=\frac{p+1}{2}$.

Now, in the next result where $q<2 p$ and $\alpha_{1}=\alpha_{1}^{\prime} q \geq 2 q$, we consider the Euclidean division of $\alpha_{2}$ by $\alpha_{1}^{\prime}: \alpha_{2}=m \alpha_{1}^{\prime}+r$ with $m \geq 1$ and $1 \leq r \leq \alpha_{1}^{\prime}-1$.

Lemma 2.11. Let $\alpha=\frac{\alpha_{1}}{\alpha_{2}} \in \mathbb{Q}-\mathcal{K S}(N)$ be such that $\operatorname{gcd}\left(\alpha_{1}, N\right)=q$. Suppose that $\alpha_{1}=\alpha_{1}^{\prime} q \geq 2 q$ and $q<2 p$. Then the following hold.

(1) $m \in\{1,2\}$.

(2) If $m=2$, then $r=1, \alpha_{1}^{\prime}=\frac{s-1}{p-s}$ and $\alpha_{2}=\frac{q-2}{p-s}$.

(3) If $m=1$, then there exist $d_{p} \in \operatorname{Div}(p-1), d_{q} \in \operatorname{Div}(q-1)$ and $\varepsilon \in\{-1,1\}$ such that $\alpha_{1}^{\prime}=\frac{p d_{p}-\varepsilon d_{q}}{s}$ and $\alpha_{2}=\frac{q d_{p}-\varepsilon d_{q}}{s}$.

Proof. (1) Let $\alpha=\frac{\alpha_{1}}{\alpha_{2}} \in \mathbb{Q}-\mathcal{K S}(N)$ be such that $\alpha_{1}=\alpha_{1}^{\prime} q, q=p+s$ and $\operatorname{gcd}\left(\alpha_{1}, p\right)=1$. Then by (2.15) we obtain

$$
\left(\alpha_{2}-\alpha_{1}^{\prime}\right) p-\alpha_{1}^{\prime} s \mid p+s-1 .
$$

Since $\alpha_{2}=m \alpha_{1}^{\prime}+r$ and $s \leq p-1$, it follows that

$$
\left((m-2) \alpha_{1}^{\prime}+r\right) p+\alpha_{1}^{\prime}(p-s)=\left(\alpha_{2}-\alpha_{1}^{\prime}\right) p-\alpha_{1}^{\prime} s \leq p+s-1<2 p .
$$

As $\alpha_{1}^{\prime}(p-s)>0$, we get $(m-2) \alpha_{1}^{\prime}+r \leq 1$, hence

$$
(m-2) \alpha_{1}^{\prime} \leq 1-r \leq 0
$$

This implies that $m \leq 2$.

(2) Assume that $m=2$. Then by (2.18) we obtain $r=1$ and so $\alpha_{2}=2 \alpha_{1}^{\prime}+1$. Therefore (2.17) becomes $p+\alpha_{1}^{\prime}(p-s) \mid p+s-1$. But since $2\left(p+\alpha_{1}^{\prime}(p-s)\right)>2 p>p+s-1$, it follows that $p+\alpha_{1}^{\prime}(p-s)=p+s-1$. Hence $\alpha_{1}^{\prime}=\frac{s-1}{p-s}$ and so $\alpha_{2}=\frac{q-2}{p-s}$.

(3) Suppose that $m=1$. Then $\alpha_{2}=\alpha_{1}^{\prime}+r$ with $1 \leq r \leq \alpha_{1}^{\prime}-1$. Therefore $\left(S_{7}\right)$ becomes

$$
\left\{\begin{array}{r|r}
r p-\alpha_{1}^{\prime} s & q-1 \\
r & p-1
\end{array}\right.
$$


So, $\alpha \in \mathbb{Q}$ - $\mathcal{K S}(N)$ if and only if there exist $d_{q} \in \operatorname{Div}(q-1)$, $d_{p} \in \operatorname{Div}(p-1)$ and $\varepsilon \in\{-1,1\}$ such that

$$
\left\{\begin{aligned}
r p-\alpha_{1}^{\prime} s & =\varepsilon d_{q} \\
r & =d_{p}
\end{aligned}\right.
$$

This is equivalent to $\alpha_{1}^{\prime}=\frac{p d_{p}-\varepsilon d_{q}}{s}$ and $\alpha_{2}=\frac{q d_{p}-\varepsilon d_{q}}{s}$.

The remaining case where $\operatorname{gcd}\left(\alpha_{1}, N\right)=q$ and $q>2 p$ will be treated in the following lemma.

Lemma 2.12. Let $\alpha=\frac{\alpha_{1}}{\alpha_{2}} \in \mathbb{Q}$ be such that $\operatorname{gcd}\left(\alpha_{1}, N\right)=q$. Suppose that $\alpha_{1}=\alpha_{1}^{\prime} q$ and $q>2 p$. Then, $\alpha$ is a $K_{N}$-base if and only if there exist $\varepsilon \in\{-1,1\}, d_{p} \in \operatorname{Div}(p-1)$ and $d_{q} \in \operatorname{Div}(q-1)$ such that $\alpha_{1}^{\prime}=\frac{p d_{p}-\varepsilon d_{q}}{q-p}$ and $\alpha_{2}=\frac{q d_{p}-\varepsilon d_{q}}{q-p}$.

Proof. Let $\alpha \in \mathbb{Q}-\mathcal{K S}(N)$ be such that $\operatorname{gcd}\left(\alpha_{1}, p\right)=1, \alpha_{1}=\alpha_{1}^{\prime} q$ and $q=i p+s$. Then $\left(S_{1}\right)$ is equivalent to

$$
\left\{\begin{array}{l|l}
\alpha_{2} p-\alpha_{1}^{\prime} q \mid q-1 \\
\alpha_{2}-\alpha_{1}^{\prime} \mid p-1
\end{array}\right.
$$

Let us show that $\alpha_{2}>\alpha_{1}^{\prime}$. First, it's clear by $(2.20)$ that $\alpha_{2} \neq \alpha_{1}^{\prime}$. Now, suppose by contradiction that $\alpha_{2}<\alpha_{1}^{\prime}$. Then by (2.19) we have $\alpha_{1}^{\prime} q-\alpha_{2} p \leq q-1$, hence $q<\left(\alpha_{2}+1\right) q-\alpha_{2} p \leq \alpha_{1}^{\prime} q-\alpha_{2} p \leq q-1$, which impossible.

Since $\alpha_{2}-\alpha_{1}^{\prime}>0$, it follows by $\left(S_{8}\right)$ that $\alpha$ is a $K_{N}$-base if and only if there exist $d_{p} \in \operatorname{Div}(p-1), d_{q} \in \operatorname{Div}(q-1)$ and $\varepsilon \in\{-1,1\}$ such that

$$
\left\{\begin{aligned}
\alpha_{2} p-\alpha_{1}^{\prime} q & =\varepsilon d_{q} \\
\alpha_{2}-\alpha_{1}^{\prime} & =d_{p}
\end{aligned}\right.
$$

This is equivalent to $\alpha_{1}^{\prime}=\frac{p d_{p}-\varepsilon d_{q}}{q-p}$ and $\alpha_{2}=\frac{q d_{p}-\varepsilon d_{q}}{q-p}$.

Now, the case where $\operatorname{gcd}\left(\alpha_{1}, N\right)=p$ will be studied in the next two results.

Proposition 2.13. Let $\alpha=\frac{\alpha_{1}}{\alpha_{2}} \in \mathbb{Q}-\mathcal{K S}(N)$ be such that $\operatorname{gcd}\left(\alpha_{1}, N\right)=$ p. Suppose that $\alpha_{1}=\alpha_{1}^{\prime} p$. Then the following assertions hold.

(1) If $\alpha_{2}=1$ then $\alpha_{1}^{\prime} \in\{i, i+1\}$. 
(2) If $\alpha_{2} \geq 2$ then $i \alpha_{2}+1 \leq \alpha_{1}^{\prime} \leq(i+1) \alpha_{2}-1$.

Proof. Let $\alpha \in \mathbb{Q}-\mathcal{K} \mathcal{S}(N)$ be such that $\operatorname{gcd}\left(\alpha_{1}, q\right)=1, \alpha_{1}=\alpha_{1}^{\prime} p$ and $q=i p+s$. Then, $\left(S_{1}\right)$ is equivalent to

$$
\left(S_{9}\right) \quad \begin{cases}\alpha_{2}-\alpha_{1}^{\prime} & \mid i p+s-1 \\ \left(i \alpha_{2}-\alpha_{1}^{\prime}\right) p+\alpha_{2} s & p-1\end{cases}
$$

We claim that

$$
i \alpha_{2}-\alpha_{1}^{\prime} \leq 0
$$

Indeed, if is not the case, we get $\left(i \alpha_{2}-\alpha_{1}^{\prime}\right) p+\alpha_{2} s \geq p+\alpha_{2} s>p$, which contradicts (2.22). Also, we claim that

$$
\alpha_{1}^{\prime}-(i+1) \alpha_{2} \leq 0 .
$$

Indeed, if is not the case, we obtain $\left(\alpha_{1}^{\prime}-i \alpha_{2}\right) p-\alpha_{2} s=\left(\alpha_{1}^{\prime}-(i+1) \alpha_{2}\right) p+\alpha_{2}(p-s) \geq p+\alpha_{2}(p-s)>p$, which contradicts (2.22). Now, we consider the two following cases.

(1) If $\alpha_{2}=1$, then by (2.23) and (2.24) we get

$$
i \leq \alpha_{1}^{\prime} \leq i+1
$$

(2) Assume that $\alpha_{2} \geq 2$. We claim that $i \alpha_{2}-\alpha_{1}^{\prime} \neq 0$. Indeed if not, then $i \alpha_{2}=\alpha_{1}^{\prime}$. Hence $1=\operatorname{gcd}\left(\alpha_{1}, \alpha_{2}\right)=\operatorname{gcd}\left(\alpha_{1}^{\prime}, \alpha_{2}\right)=\alpha_{2} \geq 2$, which is impossible. Therefore by (2.23) we get

$$
i \alpha_{2}+1 \leq \alpha_{1}^{\prime} \text {. }
$$

Similarly, we claim that $\alpha_{1}^{\prime}-(i+1) \alpha_{2} \neq 0$. Hence by (2.24) we obtain

$$
\alpha_{1}^{\prime} \leq(i+1) \alpha_{2}-1
$$

Combining (2.25) and (2.26), we get

$$
i \alpha_{2}+1 \leq \alpha_{1}^{\prime} \leq(i+1) \alpha_{2}-1 \text {. }
$$

Lemma 2.14. Let $\alpha=\frac{\alpha_{1}}{\alpha_{2}} \in \mathbb{Q}$ be such that $\operatorname{gcd}\left(\alpha_{1}, N\right)=p$. Assume that $\alpha_{1}=\alpha_{1}^{\prime} p$. Then, $\alpha$ is a $K_{N}$-base if and only if there exist $\varepsilon \in$ $\{-1,1\}, d_{p} \in \operatorname{Div}(p-1)$ and $d_{q} \in \operatorname{Div}(q-1)$ such that $\alpha_{1}^{\prime}=\frac{q d_{q}+\varepsilon d_{p}}{q-p}$ and $\alpha_{2}=\frac{p d_{q}+\varepsilon d_{p}}{q-p}$. 
Proof. Let $\alpha \in \mathbb{Q}-\mathcal{K} \mathcal{S}(N)$ be such that $\operatorname{gcd}\left(\alpha_{1}, q\right)=1, \alpha_{1}=\alpha_{1}^{\prime} p$ and $q=i p+s$. As by (2.27) $\alpha_{1}^{\prime}=i \alpha_{2}+\alpha_{3}$ with $1 \leq \alpha_{3}<\alpha_{2}$ for $\alpha_{2} \geq 2$, (resp. with $0 \leq \alpha_{3} \leq \alpha_{2}$ for $\alpha_{2}=1$ ), $\left(S_{9}\right)$ becomes

$$
\left\{\begin{array}{l|l}
(i-1) \alpha_{2}+\alpha_{3} & \mid i p+s-1=q-1 \\
\alpha_{3} p-\alpha_{2} s & \mid p-1
\end{array}\right.
$$

It follows that, $\alpha$ is a $K_{N}$-base if and only if there exist $\varepsilon \in\{-1,1\}$, $d_{p} \in \operatorname{Div}(p-1)$ and $d_{q} \in \operatorname{Div}(q-1)$ such that

$$
\left\{\begin{aligned}
(i-1) \alpha_{2}+\alpha_{3} & =d_{q} \\
\alpha_{2} s-\alpha_{3} p & =\varepsilon d_{p}
\end{aligned}\right.
$$

This is equivalent to $\alpha_{2}=\frac{p d_{q}+\varepsilon d_{p}}{q-p}, \alpha_{3}=\frac{s d_{q}+\varepsilon(1-i) d_{p}}{q-p}$ and so $\alpha_{1}^{\prime}=\frac{q d_{q}+\varepsilon d_{p}}{q-p}$.

In the rest of this section, we will discuss the case where $p q \mid \alpha_{1}$.

Proposition 2.15. Let $\alpha=\frac{\alpha_{1}}{\alpha_{2}} \in \mathbb{Q}-\mathcal{K S}(N)$ be such that $\alpha_{1}=\alpha_{1}^{\prime \prime} p q$. Then the following assertions hold.

(1) $\alpha_{1}^{\prime \prime}<\alpha_{2}$.

(2) $q \leq 4 p-3$.

Proof. Let $\alpha=\frac{\alpha_{1}}{\alpha_{2}} \in \mathbb{Q}-\mathcal{K} \mathcal{S}(N)$ be such that $\alpha_{1}=\alpha_{1}^{\prime \prime} p q$. Then $\left(S_{1}\right)$ becomes

$$
\left\{\begin{array}{l|l}
\alpha_{2} p-\alpha_{1}^{\prime \prime} p q=\alpha_{2} p-\alpha_{1} & p(q-1) \\
\alpha_{2} q-\alpha_{1}^{\prime \prime} p q=\alpha_{2} q-\alpha_{1} & q(p-1)
\end{array}\right.
$$

which is equivalent to

$$
\left(S_{10}\right) \quad\left\{\begin{array}{l}
\alpha_{1}^{\prime \prime} q-\alpha_{2} \mid q-1 \\
\alpha_{1}^{\prime \prime} p-\alpha_{2} \mid p-1
\end{array}\right.
$$

(1) By (2.30) we have $\alpha_{1}^{\prime \prime} q-\alpha_{2} \leq q-1$. As in addition $q>1$, it follows that $\alpha_{1}^{\prime \prime}-1<\left(\alpha_{1}^{\prime \prime}-1\right) q \leq \alpha_{2}-1$ for $\alpha_{1}^{\prime \prime}>1$ which implies that $\alpha_{1}^{\prime \prime}<\alpha_{2}$. If $\alpha_{1}^{\prime \prime}=1$, then since $p q \neq \alpha=\frac{p q}{\alpha_{2}}$ with $\alpha_{2} \geq 1$ we get $\alpha_{2}>1=\alpha_{1}^{\prime \prime}$.

(2) By $\left(S_{10}\right)$ we can write

$$
\left\{\begin{array}{l|l}
\alpha_{1}^{\prime \prime} q-\alpha_{2} & \alpha_{1}^{\prime \prime}(q-1)=\alpha_{1}^{\prime \prime} q-\alpha_{2}+\alpha_{2}-\alpha_{1}^{\prime \prime} \\
\alpha_{1}^{\prime \prime} p-\alpha_{2} & p-1
\end{array}\right.
$$


hence

$$
\left\{\begin{array}{l|l}
\alpha_{1}^{\prime \prime} q-\alpha_{2} & \alpha_{2}-\alpha_{1}^{\prime \prime} \\
\alpha_{1}^{\prime \prime} p-\alpha_{2} & p-1
\end{array}\right.
$$

This implies that

$$
\left\{\begin{aligned}
\alpha_{1}^{\prime \prime} q-\alpha_{2} & \leq \alpha_{2}-\alpha_{1}^{\prime \prime} \\
-p+1 & \leq \alpha_{1}^{\prime \prime} p-\alpha_{2}
\end{aligned}\right.
$$

therefore

$$
\left\{\begin{aligned}
\frac{(q+1) \alpha_{1}^{\prime \prime}}{2} & \leq \alpha_{2} \\
\alpha_{2} & \leq\left(\alpha_{1}^{\prime \prime}+1\right) p-1
\end{aligned}\right.
$$

So, by combining (2.32) and (2.33), we get

$$
\frac{(q+1) \alpha_{1}^{\prime \prime}}{2} \leq \alpha_{2} \leq\left(\alpha_{1}^{\prime \prime}+1\right) p-1 \text {. }
$$

Hence $\alpha_{1}^{\prime \prime}(q-2 p+1) \leq 2(p-1)$. As in addition $\alpha_{1}^{\prime \prime} \geq 1$, we get $q-2 p+1 \leq \alpha_{1}^{\prime \prime}(q-2 p+1) \leq 2(p-1)$. Thus $q \leq 4 p-3$.

Lemma 2.16. Let $\alpha=\frac{\alpha_{1}}{\alpha_{2}} \in \mathbb{Q}$ be such that $\alpha_{1}=\alpha_{1}^{\prime \prime} p q$. Suppose that $2 p<q<3 p$. Then, $\alpha$ is a $K_{N}$-base if and only if one of the following assertions is verified.

(1) $q=\frac{9 p-5}{4}$ or $q=3 p-2, \alpha_{1}^{\prime \prime}=1$ and $\alpha_{2}=\frac{3 p-1}{2}$.

(2) $s+1 \mid q-1, \alpha_{1}^{\prime \prime}=1$ and $\alpha_{2}=2 p-1$.

(3) $\frac{s+1}{2} \mid p-1, \alpha_{1}^{\prime \prime}=1$ and $\alpha_{2}=\frac{q+1}{2}$.

Proof. Suppose that $\alpha \in \mathbb{Q}-\mathcal{K} \mathcal{S}(N)$. Then by (2.30) and (2.31) we get respectively

and

$$
\alpha_{1}^{\prime \prime} q-\alpha_{2} \leq q-1
$$

$$
-\alpha_{1}^{\prime \prime} p+\alpha_{2} \leq p-1
$$

Adding (2.34) to (2.35), we obtain

$$
\alpha_{1}^{\prime \prime}(q-p) \leq q+p-2 .
$$

Since $q=2 p+s$, it follows that

$$
\alpha_{1}^{\prime \prime} \leq \frac{q+p-2}{q-p}=\frac{3(p+s)-2 s-2}{p+s}<3 .
$$

Therefore, $\alpha_{1}^{\prime \prime} \in\{1,2\}$. Let us show that $\alpha_{1}^{\prime \prime}=1$. 
Suppose by contradiction that $\alpha_{1}^{\prime \prime}=2$. Then by (2.34) and (2.35) we get $2 p+s+1=q+1 \leq \alpha_{2} \leq 3 p-1$. Hence, the Euclidean division of $\alpha_{2}$ by $p$ is $\alpha_{2}=2 p+u$ with $s+1 \leq u \leq p-1$.

As by (2.30) we have $2\left(2 q-\alpha_{2}\right)-(q-1)=2(p-u)+3 s+1>0$ and $\left(2 q-\alpha_{2}\right) \mid(q-1)$, then we must have $2 q-\alpha_{2}=q-1$ that is $\alpha_{2}=q+1$. But, $2 \mid \operatorname{gcd}(q+1,2)=\operatorname{gcd}\left(\alpha_{2}, \alpha_{1}^{\prime \prime}\right)=1$, which is not true. So, $\alpha_{1}^{\prime \prime}=1$.

Now, let us show that $p<\alpha_{2} \leq 2 p-1$. As $\alpha_{1}^{\prime \prime}=1,\left(S_{10}\right)$ becomes

$$
\left(S_{11}\right) \quad\left\{\begin{array}{l}
q-\alpha_{2} \mid q-1 \\
\alpha_{2}-p \mid p-1
\end{array}\right.
$$

By (2.38), it follows immediately that $\alpha_{2} \leq 2 p-1$. Hence, we can write $\alpha_{2}=k p+u$ with $k \in\{0,1\}$ and $0<u \leq p-1$.

We claim that $k \neq 0$ (i.e. $k=1$ and so $p<\alpha_{2}$ ). Indeed, if not, then $\alpha_{2}=u$ and as $2 u<2 p<q$, we get

$$
2\left(q-\alpha_{2}\right)=q+(q-2 u) \geq q+1>q-1 .
$$

Therefore, by (2.37) we must have $q-\alpha_{2}=q-1$, so that $\alpha_{2}=1$. This implies that $\alpha=p q=N$, which is not possible by definition. Thus,

$$
p<\alpha_{2} \leq 2 p-1<q
$$

Since $q-\alpha_{2}>0$ and $\alpha_{2}-p>0$ by (2.39) it follows by $\left(S_{11}\right)$ that $\alpha$ is a $K_{N}$-base if and only if there exist $d_{p} \in \operatorname{Div}(p-1)$ and $d_{q} \in \operatorname{Div}(q-1)$ such that

$$
\left\{\begin{array}{l}
q-\alpha_{2}=d_{q} \\
\alpha_{2}-p=d_{p}
\end{array}\right.
$$

Let us show that $d_{p}=\frac{p-1}{k_{1}}$ and $d_{q}=\frac{q-1}{k_{2}}$ with $\left(k_{1}, k_{2}\right) \neq(1,1)$ and $\left(k_{1} \in\{1,2\}\right.$ or $\left.k_{2} \in\{1,2\}\right)$.

Adding (2.40) to (2.41), we get

$$
q-p=d_{q}+d_{p}
$$

- If $d_{p}=p-1$ and $d_{q}=q-1$, then by (2.42) we obtain

$$
p+s=q-p=d_{q}+d_{p}=q+p-2=3 p+s-2 .
$$

Hence $p=1$, which is not possible.

- Now, assume that $d_{p} \leq \frac{p-1}{3}$ and $d_{q} \leq \frac{q-1}{3}$. Then by (2.42) $p+s=q-p=d_{q}+d_{p} \leq \frac{p+q-2}{3}$. Hence $2 s \leq-2$, which is not true. 
So, we conclude that $d_{p} \in\left\{p-1, \frac{p-1}{2}\right\}$ or $d_{q} \in\left\{q-1, \frac{q-1}{2}\right\}$. First, we claim that $d_{q} \neq q-1$, indeed if $d_{q}=q-1$ it follows by (2.40) and (2.41) that $\alpha_{2}=1$ and so $\alpha=p q=N$ which is not possible. This yields to consider the following cases:

(1) If $d_{p}=\frac{p-1}{2}$, then by (2.41) we have $\alpha_{2}=\frac{3 p-1}{2}$. Therefore $q-\alpha_{2}=\frac{p+2 s+1}{2} \mid q-1=2 p+s-1$ by (2.40). Knowing that $4\left(q-\alpha_{2}\right)-(q-1)=3 s+3>0$ and $q-\alpha_{2}-(q-1)=1-\alpha_{2}<0$, it follows that $q-1=k\left(q-\alpha_{2}\right)$ with $k \in\{2,3\}$. This implies that $k=2$ (i.e. $d_{q}=\frac{q-1}{2}$ ) hence $s=p-2$ and $q=3 p-2$ or $k=3$ (i.e. $d_{q}=\frac{q-1}{3}$ ) which gives $s=\frac{p-5}{4}$ and $q=\frac{9 p-5}{4}$.

(2) Suppose that $d_{p}=p-1$. Then by (2.41) we obtain $\alpha_{2}=2 p-1$, this yields by (2.40) that $q-\alpha_{2}=s+1 \mid q-1$.

(3) Now, assume that $d_{q}=\frac{q-1}{2}$. Then by (2.40) we obtain $\alpha_{2}=$ $\frac{q+1}{2}$, this yields by (2.41) that $\alpha_{2}-p=\frac{s+1}{2} \mid p-1$.

This ends the forward direction of the proposition. The backward direction is clearly satisfied.

Lemma 2.17. Let $\alpha=\frac{\alpha_{1}}{\alpha_{2}} \in \mathbb{Q}$ be such that $\alpha_{1}=\alpha_{1}^{\prime \prime} p q$. Suppose that $3 p<q<4 p$. Then, $\alpha$ is a $K_{N}$-base if and only if $q=4 p-3, \alpha_{1}^{\prime \prime}=1$ and $\alpha_{2}=2 p-1$.

Proof. Suppose that $\alpha \in \mathbb{Q}-\mathcal{K} \mathcal{S}(N), \alpha_{1}=\alpha_{1}^{\prime \prime} p q$ and $3 p<q<4 p$.

Let us show that $\alpha_{1}^{\prime \prime}=1$. As $q=3 p+s$ we get by (2.36)

$$
\alpha_{1}^{\prime \prime} \leq \frac{q+p-2}{q-p}=\frac{2(2 p+s)-s-2}{2 p+s}<2 \text {. }
$$

Thus, $\alpha_{1}^{\prime \prime}=1$.

Now, since $\alpha_{1}^{\prime \prime}=1$ and with the same proof of (2.39), we may write

$$
p<\alpha_{2}=p+u \leq 2 p-1<q .
$$

Hence, $\left(S_{10}\right)$ becomes

$$
\begin{cases}2 p+s-u=q-\alpha_{2} & \mid q-1=3 p+s-1 \\ u=\alpha_{2}-p & \mid p-1\end{cases}
$$


As $3(2 p+s-u)=3 p+s-1+3(p-u)+2 s+1>3 p+s-1$ and $2 p+s-u<3 p+s-1$, it follows by (2.43) that $2(2 p+s-u)=3 p+s-1$. Hence, $u=\frac{p+s+1}{2}$.

By (2.44), we have $u=\frac{p+s+1}{2} \mid(p-1)$. Since, in addition, $2 u=p+s+1>p-1$, it follows that $\frac{p+s+1}{2}=p-1$. Hence $s=p-3 ;$ so that $q=4 p-3$ and $\alpha_{2}=2 p-1$.

This ends the forward direction of the proposition. The backward direction is clearly satisfied.

Now, for the next two results, we consider $\alpha_{2}=k p+u$ with $1 \leq u \leq$ $p-1$; the Euclidian division of $\alpha_{2}$ by $p$.

Proposition 2.18. Let $\alpha=\frac{\alpha_{1}}{\alpha_{2}} \in \mathbb{Q}-\mathcal{K S}(N)$ be such that $\alpha_{1}=\alpha_{1}^{\prime \prime} p q$. If $q<2 p$, then the following assertions hold.

(1) $\alpha_{1}^{\prime \prime} \leq p+1$.

(2) $\alpha_{1}^{\prime \prime} \in\{k, k+1\}$.

Proof. Suppose that $\alpha \in \mathbb{Q}-\mathcal{K} \mathcal{S}(N)$ and $q=p+s$.

(1) By (2.36) we have $\alpha_{1}^{\prime \prime} \leq \frac{q+p-2}{q-p}=\frac{2 p+s-2}{s}$. Hence, two cases are to be considered:

- If $s=1$ and so $q=3, p=2$, then $\alpha_{1}^{\prime \prime} \leq 3=p+1$.

- If $s \geq 2$, then $\alpha_{1}^{\prime \prime} \leq \frac{2 p+s-2}{s} \leq p$.

(2) Substituting $q=p+s$ and $\alpha_{2}=k p+u$ in (2.34), we get $\left(\alpha_{1}^{\prime \prime}-k\right) p+\alpha_{1}^{\prime \prime} s-u=\alpha_{1}^{\prime \prime} q-\alpha_{2} \leq q-1=p+s-1$, therefore $\left(\alpha_{1}^{\prime \prime}-k-1\right) p+\left(\alpha_{1}^{\prime \prime}-1\right) s \leq u-1 \leq p-2$. Since, in addition, $\alpha_{1}^{\prime \prime} \geq 1$ hence $\left(\alpha_{1}^{\prime \prime}-1\right) s \geq 0$, it follows that $\alpha_{1}^{\prime \prime}-k-1 \leq 0$, that is $\alpha_{1}^{\prime \prime} \leq k+1$.

Also, by (2.35) we have $\left(\alpha_{1}^{\prime \prime}-k\right) p-u=\alpha_{1}^{\prime \prime} p-\alpha_{2} \geq-p+1$, hence $\left(\alpha_{1}^{\prime \prime}-k+1\right) p \geq u+1 \geq 2$. Therefore, $\alpha_{1}^{\prime \prime}-k+1 \geq 1$, that is $\alpha_{1}^{\prime \prime} \geq k$. So, we deduce that $\alpha_{1}^{\prime \prime} \in\{k, k+1\}$.

Now, the next two lemmas deal with the two cases of $\alpha_{1}^{\prime \prime} \in\{k, k+1\}$.

Lemma 2.19. Let $\alpha=\frac{\alpha_{1}}{\alpha_{2}} \in \mathbb{Q}$ be such that $\alpha_{1}=k p q$ and $q<2 p$. Then, $\alpha$ is a $K_{N}$-base if and only if there exist $\varepsilon \in\{-1,1\}, d_{p} \in$ 
$\operatorname{Div}(p-1)$ and $d_{q} \in \operatorname{Div}(q-1)$ such that $k=\frac{d_{p}+\varepsilon d_{q}}{s}>0$ and $\alpha_{2}=\frac{q d_{p}+\varepsilon p d_{q}}{s}>0$.

Proof. Replacing $\alpha_{2}=k p+u, q=p+s$ and $\alpha_{1}=k p q$ in $\left(S_{10}\right)$, we obtain

$$
\left\{\begin{array}{r|r}
k s-u & q-1 \\
-u & p-1
\end{array}\right.
$$

Hence, $\alpha$ is a $K_{N}$-base if and only if there exist $d_{q} \in \operatorname{Div}(q-1)$, $d_{p} \in \operatorname{Div}(p-1)$ and $\varepsilon \in\{-1,1\}$ such that

$$
\left\{\begin{aligned}
k s-u & =\varepsilon d_{q} \\
u & =d_{p}
\end{aligned}\right.
$$

This is equivalent to $k=\frac{d_{p}+\varepsilon d_{q}}{s}$ and $\alpha_{2}=\frac{q d_{p}+\varepsilon p d_{q}}{s}$.

Lemma 2.20. Let $\alpha=\frac{\alpha_{1}}{\alpha_{2}} \in \mathbb{Q}$ be such that $\alpha_{1}=(k+1) p q$ and $q<2 p$. Then, $\alpha$ is a $K_{N}$-base if and only if there exist $d_{p} \in \operatorname{Div}(p-1)$ and $d_{q} \in \operatorname{Div}(q-1)$ such that $k+1=\frac{d_{q}-d_{p}}{s}>0$ and $\alpha_{2}=\frac{p d_{q}-q d_{p}}{s}>0$.

Proof. Substituting $\alpha_{2}=k p+u, q=p+s$ and $\alpha_{1}=(k+1) p q$ in $\left(S_{10}\right)$, we get

$$
\left\{\begin{array}{r|r}
(k+1) s+p-u & q-1 \\
p-u & p-1
\end{array}\right.
$$

Therefore, $\alpha$ is a $K_{N^{-}}$base if and only if there exist $d_{p} \in \operatorname{Div}(p-1)$ and $d_{q} \in \operatorname{Div}(q-1)$ such that

$$
\left\{\begin{aligned}
(k+1) s+p-u & =d_{q} \\
p-u & =d_{p}
\end{aligned}\right.
$$

This is equivalent to $k+1=\frac{d_{q}-d_{p}}{s}>0$ and $\alpha_{2}=\frac{p d_{q}-q d_{p}}{s}>0$.

\section{The Korselt Set of $p q$}

First, let us give the Korselt set of $p q$ over $\mathbb{Z}$ when $q<2 p$.

Corollary 3.1. Let $\alpha \in \mathbb{Z}$ such that $\operatorname{gcd}(\alpha, N)=1$. Assume that $q<2 p$. Then, $\alpha$ is a $K_{N}$-base if and only if there exist $d_{q} \in \operatorname{Div}(q-1)$ and $\varepsilon \in\{-1,1\}$ such that $\alpha=p+\varepsilon d_{q}$ and $s-\varepsilon d_{q}$ divides $p-1$. 
Proof. Let $\alpha \in \mathbb{Z}$ (i.e. $\alpha_{2}=1$ ) such that $\operatorname{gcd}(\alpha, N)=1$. Suppose that $q<2 p$. Then, we have by Lemmas 2.6, 2.7 and 2.8 , respectively, $\alpha$ is a $K_{N}$-base if and only if

- $\alpha=q+p-1$,

- $\alpha=p+d_{q}$ such that $\left(s-d_{q}\right) \mid(p-1), d_{q} \leq p-1$.

- $\alpha=p-d_{q}>0$ such that $\left(s+d_{q}\right) \mid(p-1)$.

This is equivalent to the existence of $d_{q} \in \operatorname{Div}(q-1)$ and $\varepsilon \in\{-1,1\}$ such that $\alpha=p+\varepsilon d_{q}>0$ and $\left(s-\varepsilon d_{q}\right) \mid(p-1)$.

By Corollary 3.1, we derive immediately the following.

Theorem 3.2. Let $p<q$ be two prime numbers, $N=p q$ and $q=p+s$ such that $1 \leq s \leq p-1$. Then

$\mathbb{Z}-\mathcal{K S}(N)=\left(\bigcup_{\substack{d_{q} \mid q-1 \\ \varepsilon \in\{-1,1\}}}\left\{p+\varepsilon d_{q} ; s-\varepsilon d_{q} \mid p-1\right\}\right) \cup\{2 p ; p-s \mid p-1\}$.

Now, before giving the mean result in this paper, we set for given distinct prime numbers $p$ and $q$ the following:

$$
\begin{aligned}
& \mathcal{A}_{p, q}=\bigcup_{\substack{d_{q}\left|q-1, d_{p}\right| p-1 \\
\varepsilon \in\{-1,1\}}}\left\{\frac{q d_{q}+\varepsilon p d_{p}}{d_{q}+\varepsilon d_{p}} ;(q-p) \mid\left(d_{q}+\varepsilon d_{p}\right), d_{q}+\varepsilon d_{p} \neq 0\right\} \\
& \mathcal{B}_{p, q}=\bigcup_{\substack{d_{q}\left|q-1, d_{p}\right| p-1 \\
\varepsilon \in\{-1,1\}}}\left\{\frac{\left(p d_{p}-\varepsilon d_{q}\right) q}{q d_{p}-\varepsilon d_{q}} ;(q-p) \mid\left(q d_{p}-\varepsilon d_{q}\right), q-p<p d_{p}-\varepsilon d_{q}\right\} \\
& \mathcal{C}_{p, q}=\bigcup_{\substack{d_{q}\left|q-1, d_{p}\right| p-1 \\
\varepsilon \in\{-1,1\}}}\left\{\frac{\left(q d_{q}+\varepsilon d_{p}\right) p}{p d_{q}+\varepsilon d_{p}} ;(q-p) \mid\left(p d_{q}+\varepsilon d_{p}\right)\right\} \\
& \mathcal{D}_{p, q}=\bigcup_{\substack{d_{q}\left|q-1, d_{p}\right| p-1 \\
\varepsilon \in\{-1,1\}}}\left\{\frac{\left(d_{p}+\varepsilon d_{q}\right) p q}{q d_{p}+\varepsilon p d_{q}} ;(q-p) \mid\left(d_{p}+\varepsilon d_{q}\right), d_{p}+\varepsilon d_{q} \neq 0\right\}
\end{aligned}
$$

Theorem 3.3 (Structure of the Rational Korselt Set of $p q$ ). Let $p<q$ be two prime numbers, $N=p q$ and $q=i p+s$ such that $1 \leq s \leq p-1$. Then the following properties hold. 
(1) If $q>4 p$, then

$$
\mathbb{Q}-\mathcal{K} \mathcal{S}(N)=\mathcal{B}_{p, q} \cup \mathcal{C}_{p, q} \cup\{p+q-1\} .
$$

(2) Assume that $3 p<q<4 p$. Then the following assertions hold.

(a) If $q=4 p-3$, then

$\mathbb{Q}-\mathcal{K} \mathcal{S}(N)=\mathcal{B}_{p, q} \cup \mathcal{C}_{p, q} \cup\left\{q-p+1, p+q-1, \frac{p q}{2 p-1}\right\}$.

(b) If $q \neq 4 p-3$, then

$$
\mathbb{Q}-\mathcal{K} \mathcal{S}(N)=\mathcal{B}_{p, q} \cup \mathcal{C}_{p, q} \cup\{p+q-1\} .
$$

(3) Suppose that $2 p<q<3 p$. Then the following conditions are satisfied.

(i) If $s+1$ divides $q-1$.

(a) If $s=\frac{p-5}{4}$ or $s=p-2$, then

$\mathbb{Q}-\mathcal{K} \mathcal{S}(N)=\mathcal{B}_{p, q} \cup \mathcal{C}_{p, q} \cup\left\{3 q-5 p+3, q-p+1, p+q-1, \frac{2 p+q-1}{2}, \frac{2 p q}{3 p-1}, \frac{p q}{2 p-1}, \frac{2 p q}{q+1}\right\}$.

(b) If $s \neq \frac{p-5}{4}$ and $s \neq p-2$ then

$\mathbb{Q}-\mathcal{K S}(N)=\mathcal{B}_{p, q} \cup \mathcal{C}_{p, q} \cup\left\{q-p+1, p+q-1, \frac{2 p+q-1}{2}, \frac{p q}{2 p-1}, \frac{2 p q}{q+1}\right\}$.

(ii) If $s+1$ not dividing $q-1$, then

$$
\mathbb{Q}-\mathcal{K S}(N)=\mathcal{B}_{p, q} \cup \mathcal{C}_{p, q} \cup\{p+q-1\} .
$$

(4) Assume that $q<2 p$. Then the following assertions hold.

(i) If $q=5$, then

$$
\mathbb{Q}-\mathcal{K} \mathcal{S}(N)=\mathcal{A}_{p, q} \cup \mathcal{B}_{p, q} \cup \mathcal{C}_{p, q} \cup \mathcal{D}_{p, q} \cup\left\{\frac{q}{2}, \frac{q}{3}\right\} .
$$

(ii) Suppose that $q \neq 5$. Then the following subcases hold.

(a) If $p>2$ and $s=\frac{p+1}{2}$, then

$$
\mathbb{Q}-\mathcal{K} \mathcal{S}(N)=\mathcal{A}_{p, q} \cup \mathcal{B}_{p, q} \cup \mathcal{C}_{p, q} \cup \mathcal{D}_{p, q} \cup\left\{\frac{q}{3}\right\} .
$$

(b) If $s=p-1$, then

$$
\mathbb{Q}-\mathcal{K} \mathcal{S}(N)=\mathcal{A}_{p, q} \cup \mathcal{B}_{p, q} \cup \mathcal{C}_{p, q} \cup \mathcal{D}_{p, q} \cup\left\{\frac{(s-1) q}{q-2}, \frac{q}{2}\right\} .
$$

(c) Assume $p-s$ divides $2 s-1$ and $s \neq p-1$. Then

$$
\mathbb{Q}-\mathcal{K} \mathcal{S}(N)=\mathcal{A}_{p, q} \cup \mathcal{B}_{p, q} \cup \mathcal{C}_{p, q} \cup \mathcal{D}_{p, q} \cup\left\{\frac{q}{2}\right\} .
$$


(d) If $p-s$ divides $s-1, s \neq \frac{p+1}{2}$ and $s \neq p-1$, then $\mathbb{Q}-\mathcal{K} \mathcal{S}(N)=\mathcal{A}_{p, q} \cup \mathcal{B}_{p, q} \cup \mathcal{C}_{p, q} \cup \mathcal{D}_{p, q} \cup\left\{\frac{(s-1) q}{q-2}\right\}$.

(e) If $p-s$ divides neither $s-1$ nor $2 s-1$, then $\mathbb{Q}-\mathcal{K} \mathcal{S}(N)=\mathcal{A}_{p, q} \cup \mathcal{B}_{p, q} \cup \mathcal{C}_{p, q} \cup \mathcal{D}_{p, q}$.

Proof. For $N=p q$, let the sets

$$
\begin{aligned}
& \mathcal{A}=\left\{\alpha=\frac{\alpha_{1}}{\alpha_{2}} \in \mathbb{Q}-\mathcal{K} \mathcal{S}(N) ; \operatorname{gcd}\left(\alpha_{1}, N\right)=1\right\}, \\
& \mathcal{B}=\left\{\alpha=\frac{\alpha_{1}}{\alpha_{2}} \in \mathbb{Q}-\mathcal{K} \mathcal{S}(N) ; \operatorname{gcd}\left(\alpha_{1}, N\right)=q\right\}, \\
& \mathcal{C}=\left\{\alpha=\frac{\alpha_{1}}{\alpha_{2}} \in \mathbb{Q}-\mathcal{K} \mathcal{S}(N) ; \operatorname{gcd}\left(\alpha_{1}, N\right)=p\right\}, \\
& \mathcal{D}=\left\{\alpha=\frac{\alpha_{1}}{\alpha_{2}} \in \mathbb{Q}-\mathcal{K} \mathcal{S}(N) ; \operatorname{gcd}\left(\alpha_{1}, N\right)=p q\right\} .
\end{aligned}
$$

Clearly, we have

$$
\mathbb{Q}-\mathcal{K} \mathcal{S}(N)=\mathcal{A} \cup \mathcal{B} \cup \mathcal{C} \cup \mathcal{D} \text {. }
$$

First, note that if $q>2 p$, then by Lemmas 2.12 and 2.14 we have respectively $\mathcal{C}=\mathcal{C}_{p, q}$ and $\mathcal{B}=\mathcal{B}_{p, q}$. Also, by Proposition 2.4

$$
\mathcal{A}=\{\alpha \in \mathbb{Z}-\mathcal{K} \mathcal{S}(N) ; \operatorname{gcd}(\alpha, N)=1\} .
$$

(1) If $q>4 p$, then we have by [4, Theorem 14] and Proposition 2.15, respectively, $\mathcal{A}=\{q+p-1\}$ and $\mathcal{D}=\emptyset$. Therefore,

$$
\mathbb{Q}-\mathcal{K} \mathcal{S}(N)=\mathcal{B}_{p, q} \cup \mathcal{C}_{p, q} \cup\{q+p-1\} .
$$

(2) Assume that $3 p<q<4 p$. Then by [4, Theorem 14] we consider the following two subcases.

(a) Suppose that $q=4 p-3$. Then $\mathcal{A}=\{q-p+1, q+p-1\}$. Since $\mathcal{D}=\left\{\frac{p q}{2 p-1}\right\}$ by Lemma 2.17 it follows that $\mathbb{Q}-\mathcal{K S}(N)=\mathcal{B}_{p, q} \cup \mathcal{C}_{p, q} \cup\left\{q-p+1, p+q-1, \frac{p q}{2 p-1}\right\}$.

(b) If $q \neq 4 p-3$, then $\mathcal{A}=\{q+p-1\}$. Also, since $\mathcal{D}=\emptyset$ by Lemma 2.17 we deduce that

$$
\mathbb{Q}-\mathcal{K} \mathcal{S}(N)=\mathcal{B}_{p, q} \cup \mathcal{C}_{p, q} \cup\{p+q-1\} .
$$

(3) Now, suppose that $2 p<q<3 p$. Then by Lemma 2.16 several subcases are to be discussed. 
(i) If $s+1$ divides $q-1$ (which is equivalent to $\frac{s+1}{2}$ divides $p-1)$.

(a) If $s=\frac{p-5}{4}$ or $s=p-2$, then $\mathcal{D}=\left\{\frac{2 p q}{3 p-1}, \frac{p q}{2 p-1}, \frac{2 p q}{q+1}\right\}$ and by [4, Lemma 12],

$$
\mathcal{A}=\left\{3 q-5 p+3, q-p+1, p+q-1, \frac{2 p+q-1}{2}\right\} .
$$

Thus, we conclude that

$$
\mathbb{Q}-\mathcal{K} \mathcal{S}(N)=\mathcal{B}_{p, q} \cup \mathcal{C}_{p, q} \cup\left\{3 q-5 p+3, q-p+1, p+q-1, \frac{2 p+q-1}{2}, \frac{2 p q}{3 p-1}, \frac{p q}{2 p-1}, \frac{2 p q}{q+1}\right\} \text {. }
$$

(b) If $s \neq \frac{p-5}{4}$ and $s \neq p-2$, then $\mathcal{D}=\left\{\frac{p q}{2 p-1}, \frac{2 p q}{q+1}\right\}$.

Now, let $\mathcal{S}=\left\{q-p+1, p+q-1, \frac{2 p+q-1}{2}\right\}$. We

will prove that $\mathcal{A}=\mathcal{S}$.

Since $s+1 \mid q-1$, it follows by definition that $\mathcal{S} \subseteq \mathcal{A}$.

Also, as $s \neq \frac{p-5}{4}$, it follows according to [4, Lemma 12], that $\mathcal{A} \subseteq \mathcal{S}$ and so $\mathcal{A}=\mathcal{S}$. Thus,

$$
\mathbb{Q}-\mathcal{K} \mathcal{S}(N)=\mathcal{B}_{p, q} \cup \mathcal{C}_{p, q} \cup\left\{q-p+1, p+q-1, \frac{2 p+q-1}{2}, \frac{p q}{2 p-1}, \frac{2 p q}{q+1}\right\} \text {. }
$$

(ii) Now, suppose that $s+1$ doesn't divide $q-1$. Then $\mathcal{D}=\emptyset$ and by [4, Lemma 12] we have $\mathcal{A}=\{p+q-1\}$. So,

$$
\mathbb{Q}-\mathcal{K} \mathcal{S}(N)=\mathcal{B}_{p, q} \cup \mathcal{C}_{p, q} \cup\{p+q-1\} \text {. }
$$

(4) Assume that $q<2 p$. Then by Lemmas 2.6, 2.7 and 2.8 we have $\mathcal{A}=\mathcal{A}_{p, q}$. Further, $\mathcal{C}=\mathcal{C}_{p, q}$ by Lemma 2.14 and by lemmas 2.19 and 2.20 we have $\mathcal{D}=\mathcal{D}_{p, q}$.

Now, let us determine the set $\mathcal{B}$. First, we remark by Lemma 2.11 and Proposition 2.10 that

$$
\mathcal{B}_{p, q} \subseteq \mathcal{B} \subseteq \mathcal{B}_{p, q} \cup\left\{\frac{(s-1) q}{q-2}, \frac{q}{2}, \frac{q}{3}\right\} .
$$

Also, we need to state the next four assertions which can be verified easily, in order to determine completely $\mathcal{B}$.

- If $\frac{q}{3} \in \mathcal{B}$ then $\frac{(s-1) q}{q-2} \in \mathcal{B}$.

- $\left\{\frac{(s-1) q}{q-2}, \frac{q}{2}\right\} \subseteq \mathcal{B}$ if and only if $s=p-1$. 
- $\left\{\frac{q}{2}, \frac{q}{3}\right\} \subseteq \mathcal{B}$ if and only if $q=5$.

- If $q=5$ then $\frac{(s-1) q}{q-2}=\frac{q}{3}$.

This leads us to consider the following cases.

(i) If $q=5$, then $\mathcal{B}=\mathcal{B}_{p, q} \cup\left\{\frac{q}{2}, \frac{q}{3}\right\}$. Thus,

$$
\mathbb{Q}-\mathcal{K S}(N)=\mathcal{A}_{p, q} \cup \mathcal{B}_{p, q} \cup \mathcal{C}_{p, q} \cup \mathcal{D}_{p, q} \cup\left\{\frac{q}{2}, \frac{q}{3}\right\}
$$

(ii) Suppose that $q \neq 5$. Then the following subcases hold.

(a) If $p>2$ and $s=\frac{p+1}{2}$, then $\frac{q}{3} \in \mathcal{B}$ and so $\frac{(s-1) q}{q-2} \in$ $\mathcal{B}$.

Since in addition $q \neq 5$, we have $\frac{q}{2} \notin \mathcal{B}$. Therefore $\mathcal{B}=\mathcal{B}_{p, q} \cup\left\{\frac{q}{3}\right\}$. This implies that

$\mathbb{Q}$ - $\mathcal{K} \mathcal{S}(N)=\mathcal{A}_{p, q} \cup \mathcal{B}_{p, q} \cup \mathcal{C}_{p, q} \cup \mathcal{D}_{p, q} \cup\left\{\frac{q}{3}\right\}$.

(b) If $s=p-1$, then $\left\{\frac{(s-1) q}{q-2}, \frac{q}{2}\right\} \subseteq \mathcal{B}$.

Since in addition $q \neq 5$, we have $\frac{q}{3} \notin \mathcal{B}$. Therefore $\mathcal{B}=\mathcal{B}_{p, q} \cup\left\{\frac{q}{2}, \frac{(s-1) q}{q-2}\right\}$. So, we deduce that

$\mathbb{Q}-\mathcal{K} \mathcal{S}(N)=\mathcal{A}_{p, q} \cup \mathcal{B}_{p, q} \cup \mathcal{C}_{p, q} \cup \mathcal{D}_{p, q} \cup\left\{\frac{(s-1) q}{q-2}, \frac{q}{2}\right\}$.

(c) Assume that $(p-s) \mid(2 s-1)$ and $s \neq p-1$. Then by Proposition 2.10 we have $\frac{q}{2} \in \mathcal{B}$. Also, as $p-s$ not dividing $s-1$ (else $s=p-1$ ), it follows that $\frac{(s-1) q}{q-2} \notin \mathcal{B}$ and as $q \neq 5$, we have $\frac{q}{3} \notin \mathcal{B}$. Consequently, we get by (3.1), $\mathcal{B}=\mathcal{B}_{p, q} \cup\left\{\frac{q}{2}\right\}$. This implies that $\mathbb{Q}-\mathcal{K} \mathcal{S}(N)=\mathcal{A}_{p, q} \cup \mathcal{B}_{p, q} \cup \mathcal{C}_{p, q} \cup \mathcal{D}_{p, q} \cup\left\{\frac{q}{2}\right\}$.

(d) If $(p-s) \mid(s-1), s \neq \frac{p+1}{2}$ and $s \neq p-1$, then we get respectively $\frac{(s-1) q}{q-2} \in \mathcal{B}, \frac{q}{3} \notin \mathcal{B}$ and $\frac{q}{2} \notin \mathcal{B}$. Hence, $\mathcal{B}=\mathcal{B}_{p, q} \cup\left\{\frac{(s-1) q}{q-2}\right\}$, therefore 


$$
\mathbb{Q}-\mathcal{K} \mathcal{S}(N)=\mathcal{A}_{p, q} \cup \mathcal{B}_{p, q} \cup \mathcal{C}_{p, q} \cup \mathcal{D}_{p, q} \cup\left\{\frac{(s-1) q}{q-2}\right\} .
$$

(e) Now, suppose that $p-s$ divides neither $s-1$ nor $2 s-1$. Then by Lemma 2.11 and Proposition 2.10 each of the rational numbers $\frac{(s-1) q}{q-2}, \frac{q}{2}$ and $\frac{q}{3}$ is not in $\mathcal{B}$. Hence, $\mathcal{B}=\mathcal{B}_{p, q}$. So, we conclude that $\mathbb{Q}-\mathcal{K} \mathcal{S}(N)=\mathcal{A}_{p, q} \cup \mathcal{B}_{p, q} \cup \mathcal{C}_{p, q} \cup \mathcal{D}_{p, q}$.

Acknowledgement. I thank the referee for his/her report improving both the presentation and the mathematical content of the paper.

\section{REFERENCES}

[1] N. G. W. H. Beeger; On composite numbers $n$ for which $a^{n-1} \equiv 1(\bmod n)$ for every a prime to $n$, Scripta Math. 16(1950), 133 - 135.

[2] K. Bouallègue, O. Echi, R. Pinch; Korselt Numbers and Sets, Int. J. Number Theory 6(2010), $257-269$.

[3] R. D. Carmichael; On composite numbers $P$ which satisfy the Fermat congruence $a^{P-1} \equiv 1(\bmod P)$, Amer. Math. Monthly 19(1912), $22-27$.

[4] O. Echi, N. Ghanmi; The Korselt Set of pq, Int. J. Number Theory. Vol.8, No.2(2012), $299-309$.

[5] N. Ghanmi; $\mathbb{Q}$-Korselt Numbers, Turk. J. Math 42(2018), 2752 - 2762.

[6] N. Ghanmi; Rationel Korselt Bases of Prime Powers, Submitted.

[7] O. Echi, Williams Numbers, C. R. Math. Acad. Sci. Soc. R. Can. 29(2007), 4147.

[8] A. Korselt; Problème chinois, L'intermediaire des Mathématiciens 6(1899), $142-143$.

(Ghanmi) (1)Preparatory Institute of Engineering Studies, Tunis UniVERSITY, TUNISIA.

(2) University College of Jammum, Department of Mathematics, Mekkah, Saudi Arabia.

E-mail address: naghanmi@uqu.edu.sa and neghanmi@yahoo.fr 